\title{
The History and Outlook of Animal Drugs Treating Asthma, Chronic Bronchitis, and Haze Episode-induced Respiratory Diseases
}

\author{
Xiumei $\mathrm{Wu}^{1,2}$, Hairong Zhao ${ }^{3}$, Junya Chen ${ }^{2}$, Rui Feng ${ }^{1}$, Chenggong $\mathrm{Li}^{2}$, Zhixue Zhang ${ }^{3}$, \\ Chenggui Zhang ${ }^{1,2, *}$, Guike $\mathrm{Li}^{3}$ and Yu Zhao ${ }^{2,3,{ }^{*}}$
}

${ }^{1}$ Yunnan Provincial Key Laboratory of Entomological Biopharmaceutical R\&D, Dali University, Dali 671000, Yunnan Province, China

${ }^{2}$ The Key Laboratory of Medical Insects and Spiders Resources for Development \& Utilization at Yunnan Province; Dali University, Dali 671000, Yunnan Province, China

${ }^{3}$ National-local Joint Engineering Research Center of Entomoceutics, Dali 671000, Yunnan Province, China

\begin{abstract}
Animal drugs have been historically applied in Chinese remedies for more than two thousands. It was reported that Chinese medical animals consisting of 1,590 species took up $12.5 \%$ of the total number of all TCM resources. Those animal drugs such as, earthworm, gecko, periostracum cicadae, and scorpios, of commonly used in China, are very remarkable and traditional for the treatment of asthma or chronic bronchitis. This review presents research advance of animal drugs possessing significant implications for the development of novel anti-asthma or chronic bronchitis drugs. The experimental studies and clinical efficacies against asthma and chronic bronchitis of animal drugs were summarized herein. Moreover, the potential utilization of animal drugs on inhibiting haze/fog induced respiratory diseases was also discussed.
\end{abstract}

Keywords: Animal drugs, asthma, chronic bronchitis, medicinal insects, respiratory disease.

\section{INTRODUCTION}

Animal drugs used in China has a rather long history. As an example, China began to use bees as early as 3,000 years ago. Farming of pearl and oyster, clinical applications of acutobin antler, musk, gelatin, etc. in China have over 2,000 years of history [1]. As reported in 2,000 years ago, the earliest extant Chinese Pharmacopeia "Shennong Bencao Jing" (Shennong's Herbal Classics) recorded animal medicines for 65 species. "Xinxiu Bencao" (The Newly Revised Materia Medica) appeared in 659 AD described 128 kinds of animal medicines; "Bencao Gangmu" (Compendium of Materia Medica) written in 1590 AD contains 460 kinds of animal medicines. "Medicinal Fauna of China" (1979 edition) collected 1,257 kinds of animal medicines. Publishing in 1979, the "The Dictionary of Traditional Chinese Medicine" containing for 5,767 species of TCM items, whilst 740 kinds of them are animal-oriented drugs [2]. The investigation result of "Traditional Chinese Medicine Resources to China Records" [3] indicated that, China has medicinal animals for 1,590 species, accounting for $12.5 \%$ of the total number of all TCM resources. Moreover, western countries also have historically used

*Address correspondence to this author at the Yunnan Provincial Key Laboratory of Entomological Biopharmaceutical R\&D, Dali University, Wanhua Road, Dali, Yunnan Province 671000, China; Tel: (+86) 872-2214251; Fax: (+86) 872-2257401; E-mails: chenggui_zcg@163.com, dryuzhao@126.com animal organ to combat disease, but only referred it as the "organ preparations" previously because of its effective components is not quite clear. Since 1820s, researchers begin to gradually understand the effective components in several animal organs, which lead to the discovery of the active substances such as insulin, thyroxine, etc. Between 1840s to 1850 s, people subsequently found the important role of adrenal cortical hormone and pituitary hormones on human body, thereafter caused the increasing of drug variety of this kind. Since 1960's, along with the technology matures upon isolation, separation and enzyme purification from organisms, a new era in the application of enzyme preparations in medicine has commenced. This class of drugs has increased to a number of 140 at 1970 's, and is still increasing. More and more preparations extracted from animal tissue, organs, glands, body fluids, secretions (toxin) and the skin, bone, horn, armour and placenta have been utilized for treatment of various diseases, which exhibit their effectiveness. This formulation is also often referred to as "animal biochemical drugs". This article mainly aims at the experimental study of animal source drugs to treat asthma and chronic bronchitis disease, while their clinical efficacies were summarized, both are to provide new ideas and methods for the research and development of new anti-asthma drugs. In addition, the description of the potential of animal drugs upon prevention Chinese from haze pollution was also discussed. 


\section{ANIMAL SOURCE DRUGS TO TREAT ASTHMA}

Asthma is a kind of chronic inflammatory airway diseases characterized by the features of airway hyperresponsiveness (AHR), chronic airway inflammation, and airway remodeling, and the patients with cough, wheezing, expiratory dyspnea, chest pain, and cough as the main symptoms [4]. Some patients may be accompanied by reversible airway obstruction, mucus production increase, eosinophil infiltration, and non-specific respiratory hypersensitivity [5]. The global prevalence of asthma was $1 \%-18 \%$, and it is estimated that the world has 300 million asthma patients [4]. Currently the front-line drugs for the treatment of asthma are glucocorticoids, which, however, can not thoroughly cure such disease. Many drugs against asthma was not sufficient and most prescription drugs possess side effects [6], thus promoting the development of alternative therapies. The investigations such as western and Asian herbs, acupuncture treatment, all kinds of body control (body manipulation), psychological therapy, homeopathic (homeopathy), research and desensitization treatment have dramatically increased over recent decades [7]. Chinese medical workers has also accomplished series of systematic research for seeking alternative medicines and folk medicines against asthma, during their researches upon the animal medicines traditionally used as traditional Chinese medicines. Researchers have found a number of animal drugs with significant efficacy which is summarized as follows:

\subsection{Periostracum Cicadae}

Periostracum Cicadae was initially recorded for clinical utilization in ancient Chinese medical book "Ming Yi Bie Lu" (Transactions of Famous Physicians) (ca. 150 AD). In traditional Chinese medicine it indicates the shedding hull of the larvae of the black grasshopper Crytotympana pustulata Fabriciousyo (Fabricius) upon feathering off (Figure 1). It was recorded as an alternative clinical drug upon asthma early at another ancient Chinese medicinal book "Yao Xing Lun" (Treatise on Property of Drug) (Tang dynasty, ca. 750 AD) [8]. The description on "Chinese Pharmacopoeia Practical Manual" recounts that Periostracum Cicadae demonstrates significant anticonvulsant, antipyretic, analgesic, relieve bronchial smooth muscle spasm, immunosuppressive and antiallergic effects, as well it plays a protective role the cell membrane of erythrocyte by improving the combining function with oxygen of the erythrocytes [9]. Zhang et al. [10] indicated that Periostracum Cicadae showed certain protective effect on histamine-induced asthma model. Another investigation confirms that Periostracum Cicadae exhibit apparent soothing asthma efficacy on an asthma model induced with histamine [11]. Xu [12] performed a treatment of acute bronchitis and cough for 64 cases by a cough granule made from Periostracum Cicadae. 59 cases were cured, while the cure rate is $92.18 \%$. The total effective rate was $95.31 \%$.

\subsection{Scorpio}

Traditional Chinese medicine refers the drying whole of Scorpion (Buthus martensii Karsch), Buthidae, Scorpiones (Figure 1). Fresh scorpion contains scorpion venom, trimethylamine, betaine, sulfuric acid, palmitic acid, stearic acid, cholesterol, ammonium salts, lecithin, and picric acid Gai (a kind of column picrate stored in the same poison venom gland with the scorpion venom) [13]. Scorpion venom is mainly composed of protein and non-protein parts [14], the former is actually a kind of protein similar with the snake neurotoxin, which can have an effect on the adrenergic nerve and may inhibit the reduction of airway caliber (bronchoconstriction), thus relieve respiratory spasm [15]. Further pharmacological studies [16] indicated that the "Scorpion-Scolopendra Mixture" can improve the airway inflammation in asthmatic model rats, while reducing the thickness of the bronchial wall and smooth muscle, reducing the proliferation of collagen fibers. Thereof the mixture showed certain improvement or delay effects on airway remodeling. Meanwhile, modern medical research suggests that early suppression of airway remodeling is a new target for bronchial asthma intervention [17], which reveals the outlook for Scorpion utilization upon the treatment of asthma diseases.

\subsection{Ants}

Ants are a complex group of organisms. Taxomomically these social animals attributed to Insecta, Hymenoptera, Formicidae. In recent years, pharmacological studies confirmed that Polyrhachis vicina Roger, PVR, possess clear efficacies of antiinflammatory, protection of liver, relieving asthma, analgesic, and therefore are clinically used for the treatment of rheumatoid arthritis, hepatitis, spermatorrhea, anemia and other diseases $[18,19]$. According to the research reports of Zhao et al. [20], the alcohol extract of $P$. vicina can antagonize acetylcholine-induced bronchial asthma and intestinal cramps, and against $\mathrm{BeCl}_{2}$-caused intestinal cramps. 

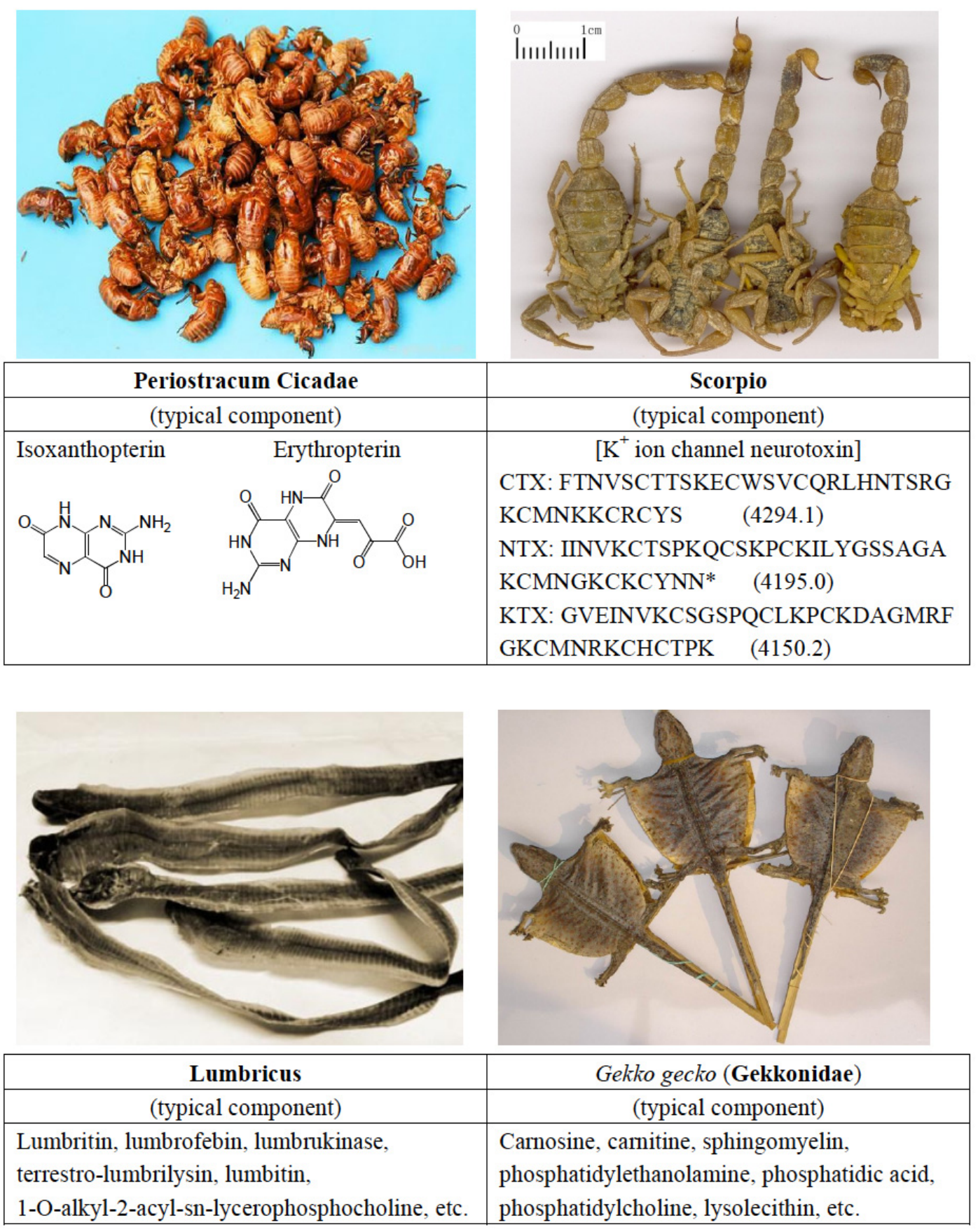

Figure 1: Typical TCM animal drugs treating asthma and chronic bronchitis, as well as their characteristic components.

Presumably the mechanism of its relieving asthma or antispasmodic effects are related with anticholinergic and direct inhibition of smooth muscle.

\subsection{Snake Bile}

Snake bile is a commonly used Chinese medicine, first recorded in "Ming Yi Bie Lu" (Transactions of Famous Physicians) (ca. 150 AD), means the gallbladders of Elapidae species such as cobra Naja naja Linnaeus, Gold-banded krait Bungarus fasciatus Schneider, or Colubridae species squirrel snakes Ptyas korros Schlegel. According to reports, an admixture made from snake bile, bezoar, and Fritillaria cirrhosa Don. can not only attenuate guinea pig asthma induced by histamine and acetylcholine mixture, but also reduce the number of animals developing asthma [21]. Cai et al. [22] compared the pharmacological efficacy of "Shedan Chuanbei Pulvis" (made from 5/6 of snake bile and $1 / 6$ of Fritillaria unibracteata Hsiao et K. C. Hsia) and "Shedan Hubei Pulvis" (made from $5 / 6$ of snake bile and 1/6 of Fritillaria hupehensis Hsiao et K. C. Hsia) and found both formulation exhibited 
strong relieving activity against asthma $(P<0.05)$. Zhang et al. [23] treated the bronchitis children with "Shedan Zhuli Mixture" (made from snake bile and bamboo juice etc.) for 82 cases, among which 62 cases were cured, 18 cases improved, 2 cases invalid, while the total efficiency was $97.5 \%$.

\subsection{Snake Venom}

Ahylysantinfarctase is a new anticoagulant and thrombolytic agents isolated from snake venom with arginase as the main component. Liu et al. [24] conduced clinical study on 22 patients with bronchial asthma. The patients were randomly divided into treatment group (12 cases administered with ahylysantinfarctase $0.5 \mathrm{U} / \mathrm{d}$ intravenous infusion for two weeks) and positive control group (10 cases, inhaled corticosteroid for two weeks) for comparison. The conventional treatments of two groups are the same, including aminophylline, antibiotics treatment for two weeks. The results showed that 9 cases of the treatment group were cured, the average cure days is $5.9 \mathrm{~d} ; 7$ cases in the control group were cured with the average cure days of $11.5 \mathrm{~d}$; both groups have 2 cases improved, and 1 case invalid, respectively. The effective rates of the two groups showed no statistical significance, but the number of cure days in the treatment group was significantly shorter than that of the control group, whilst the adverse effects of ahylysantinfarctase group is smaller than corticosteroids group volunteers.

\subsection{Placenta Hominis}

Placenta hominis is dried product of healthy human placents, contained in the ancient Chinese "Supplement to Materia Medica" (Ben Cao Shi Yi) (741 AD). Lü et al. [25] reported that the therapeutic use of placenta hominis against 34 bronchial asthma patients at the remission period, including 12 cases of bronchial asthma accompanied with chronic bronchitis and 3 cases complicated with of pulmonary heart disease, and the total efficiency is $88.2 \%$. Jiang et al. [26] taking traditional therapeutic methods combined treatment with placenta hominis upon 64 cases of asthma children, after a 2-year follow-up after treatment observation, the therapy were effective on all treated patients while their improvement rate is $57.15 \%$.

\subsection{Bombyx Batryticatus}

Bombyx batryticatus means death body of insects Bombyx mori Linnaeus., Saturniidae, Lepidoptera. After their fourth molt into the domancy state these 4-5 instar larvae were infected or artificial inoculated with Beauveria bassiana Bals Vuillant to death before harvest. Bombyx batryticatus contain $67.44 \%$ of protein, $4.38 \%$ of fat, while the protein has a role in stimulating the adrenal cortex [27], which can enhance the body's defense capabilities and regulatory functionalities. In addition, Bombyx batryticatus also contain quercetin and kaempferol, whilst quercetin has good effects of expectorant, cease cough, and have certain efficacy of relieving asthma [28]. Li [29] using Bombyx batryticatus in compatibility with insects such as scorpion, earthworm, cicada and other drugs upon clinical treatment of cough-variant asthma for 42 cases, and obtained satisfactory results. Among which 17 cases were clinical controlled, 14 cases markedly improved, 8 cases effective, 3 cases ineffective, and the total efficiency of $92.9 \%$; while the positive control group administered $0.025 \mathrm{mg}$ (2 times/d) of procaterol hydrochloride tablets (Otsuka Pharmaceutical Co., Ltd.) on 40 patients, 4 cases were clinical controlled, 12 cases markedly effective, 10 cases improved, 14 cases ineffective, and the total efficiency was $65.0 \%$. The comparison between two groups has a significant difference $(P<0.01)$.

\subsection{Bee Venom}

Bee venom refers to the defensive venom secreted by Apis mellifera Linnaeus, $A$. florea Fabricius, $A$. dorsata Fabricius, $A$. cerana Fabricius, $A$. andreniformis Smith, and $A$. laboriosa Smith. Such secreted venom via the poison glands and accessory glands of the worker bees exhibited as a transparent liquid with aromatic odor, usually stored in the poison sac, and was discharged from the stinger upon sting. It has been proved to possess several peptide and proteins, some enzymes, histamines, acids, amino acids, and trace elements [30]. Scientists have performed broad research upon the major component of bee venom [31] such as phospholipase A2, hyaluronidase, melittin, apamin, mast cell degranulation peptides and secapin. It has reported that 280 cases of asthma patients were conducted on bee stings and bee venom injection. The results show a satisfactory therapeutic effect, since the therapy can stop asthma attacks, attenuate breathing difficulties, and all test patients conscious with bee venom has expectorant effect $[31,32]$. The long-term effective rate of bee stings and bee venom injection therapy attached $80 \%$. Long-term clinical practice has proved that bee venom treatment of bronchial asthma and other allergic diseases should take a light dosage, and bee venom 
therapy is more effective against simple asthma and pediatric asthma than adult patients with complications [30-32].

\section{ANIMAL MEDICINE IN TREATING CHRONIC BRONCHITIS}

Chronic bronchitis is caused by infectious or noninfectious factors inducing a kind of chronic nonspecific inflammation attacking tracheobronchial mucosa and surrounding tissues. As it often occurs in the elderly, therefore is commonly known as the old chronic bronchitis. The pathology is characterized by bronchial wall edema, bronchial gland hyperplasia, increased mucus secretion, and smooth muscle spasm. Because of recurrent respiratory tract infections, the patient suffers long-term hypoxia, declined immune function, leading to recurrent attacks, and therefore hard to be cured whilst has a difficult recovery [33]. According to statistics, in the Chinese elderly over 50 years the incidence rate is of about 15 $\%$ to $30 \%$. As the number of patients with chronic bronchitis increased in recent years, this recurring illness keep bringing a more serious impact on the patient's daily life [34]. This paper also summarized the clinical and pharmacological studies of some animal medicines upon treatment to old chronic bronchitis, in order to provide a broader theoretical foundation for the R\&D of new animal drugs against old chronic bronchitis.

\subsection{Clamshell}

In TCM, clamshell indicated the shell of Meretrix meretrix Linnaeus or Cyclina sinensis Gmelin (Veneridae) which were harvested and dried as medicine. Deng et al. [35] reported that $20 \mathrm{~g}$ powder clamshells mixed $10 \mathrm{~g}$ of indigo is effective on the treatment of chronic bronchitis, cough and phlegm (per serving $5 \mathrm{~g}, 2$ times a day).

\subsection{Pillbugs}

Pillbug in TCM indicates dried beetles of Armadillidium vulgare Latreille or Porcellio scaber Latreille. According to clinical reports [36], 247 cases of chronic bronchitis were taken pillbug as treatment which obtained a good curative effect; recent cured in 24 cases, effective in 71 cases, improved in 107 cases, and 45 cases ineffective, the total effective rate was $81.8 \%$. For asthmatic old chronic bronchitis the total efficiency was $83.4 \%$, while to the simplex old chronic bronchitis patients the total efficiency was $79.4 \%$. The pillbugs demonstrated better effects for those patients of deficiency of kidney-YANG and cold type, which especially has some effects against their dyspnea, cough, and sputum [36].

\subsection{Acutobin}

Acutobin is a purified thrombin-like enzyme isolated from the venom of Agkistrodon acutus Guenther (Crotalinae, Viperidae) [37]. Qin Rong [38] reported that the application of acutobin injection with antibiotic therapy in 30 cases of patients with acute exacerbation of chronic bronchitis and received satisfactory results: after 5 days' treatment, markedly effective in 20 cases, 8 cases effective, 2 cases ineffective, while 1 case of 80 -year-old patient died due to pulmonary heart disease.

\section{DRUGS OF ANIMAL ORIGIN POSSESSING CURATIVE EFFECTS BOTH ON ASTHMA AND CHRONIC BRONCHITIS}

Many animals have been recorded to be ethnomedicinal drugs to treat both asthma and old chronic bronchitis, such as earthworm, gecko, cantharidin, and pearls. The following are brief introduction of their clinical efficacy and pharmacodynamic study results.

\subsection{Lumbricus}

\subsubsection{Pharmacodynamic Study Overview Upon Lumbricus}

Lumbricus (Earthworm) in TCM means the dry bodies of Annelida, Megascolecidae animals such as Pheretima aspergilum E. Perrier, Pheretima vulgaris Chen, Pheretima guillelmi Michaelsen, or Pheretima pectinifera Michaelsen, which all above owns a Chinese name of "Di Long" (Figure 1). Earliest records of clinical use of Lumbricus appeared in the "Shen Nong's Herbal Classic" (Shen Nong Ben Cao Jing) with a broad effectiveness. At Ming dynasty (1578 AD) Li Shi Zhen recorded in the "Compendium of Materia Medica" (Ben Cao Gang Mu) that the earthworms have certain effect against asthma [39]. In the past three decades, pharmacological studies and chemical composition investigations of Lumbricus have made great achievements and significant progress [40-42] Modern pharmacology studies have shown that the earthworms display various effects such as antibacterial and anti-inflammatory [43], antioxidant [44], anticoagulants [45], anti-tumor [46], cease cough and asthma, and reduce blood pressure, which is therefore popular clinically applied in the treatment of cerebral thrombosis, hypertension, bronchial asthma 
burns, and ulcers. In addition, more in-depth research of this animal drug have accomplished in the treatment of respiratory diseases.

\subsubsection{Experimental Study of Earthworm in Asthma Treatment and Clinical Efficacy Observation}

Zhou et al. [47] investigated the anti-inflammatory and anti-allergic effects of the earthworm acidic extract with allergic asthma in mice model, and the results suggested that earthworm acid part can inhibit the levels eosinophil (EOS) in bronchoalveolar lavage fluid (BALF). It can also regulate Th1/Th2 balance which plays an anti-inflammatory and anti-allergic effects in mouse allergic asthma model, while more action mechanisms needs further experimental study. Wu et al. [48] reported that the earthworm injections can relieve bronchospasm in asthmatic rats model, reducing airway resistance and improving pulmonary function. Earthworm can reduce the total number of cells, albumin and leukotriene levels in the BALF of allergic asthma guinea model; particular inhibited the increase of eosinophil (EOS), and prevents this cell from activation, since the activated EOS will release variety of toxic proteins which can directly damage epithelial [49]. Yuan et al. [50] found the Earthworm capsule ("Dilong Capsule") can improve the expression of C-JUNmRNA in the treatment of asthma; while studies have shown that the expression of C-JUN is an early marker of inflammatory cell activation and was related with inflammatory cell synthesis, and the secretions of cytokines and inflammatory mediators [51]. Ding [52] applied Earthworm Erchen Decoction (a polyjuice potion including earthworm) in treatment of cough variant asthma, the treatment group (40 patients) were cured in 25 cases, effective in 9 cases, 4 cases effective, 2 cases ineffective, while the total efficiency was $95.0 \%$; in the control group (40 patients, administered methoxyphenamine hydrochloride and Noscapine, aminophylline, and brompheniramine maleate complex) were cured 17 cases, markedly effective in 7 cases, effective in 6 cases, 10 cases ineffective, and the total efficiency was $75.0 \%$; the total efficiency of the two groups were significantly different $(P<0.05)$

\subsubsection{Earthworm Treatment of Chronic Bronchitis}

Earthworm is not only effective in the treatment of bronchial asthma, but its efficacy upon chronic bronchitis is also quite satisfactory. Pharmacological studies have shown that, earthworm can antagonize histamine and pilocarpine, and is able to enhance the anti-allergic effects. However, in the treatment of chronic bronchitis, earthworm was more often utilized in a compatibility prescription mode. Yang et al. [53] used "Huoxue Tongluo Decoction" in the treatment of chronic bronchitis and achieved good results. The researchers found that the earthworm has a significant role in relaxation of bronchial, and can antagonize histamine and pilocarpine contraction of the bronchi. Zhu [54] used "Xiaoqinglong Decoction" adding earthworm in the treatment of 100 cases of acute exacerbation chronic bronchitis patients, the results indicated that 49 cases were markedly improved (wheezing cough and expectoration disappeared), 41 cases improved (wheezing cough and expectoration were apparently relieved), 10 cases ineffective (wheezing cough and expectoration showed no improvement); the total effective rate was $90 \%$.

\subsection{Gecko}

In TCM gecko means dry body of Gekko gecko Linnaeus (Gekkonidae) with a Chinese name of "Gejie" (Figure 1), which has curative effects such as antitumor, asthma, hormone-like, and anti-inflammatory, etc. Gecko could significantly prolong the incubation period of asthma in guinea pigs, effectively inhibit their asthmatic reaction, thus indicating that Gecko has a significant role in asthma [55]. Li [56] gave Gecko antiasthma pills (Gejie Fangchuan Pills) to 70 patients with bronchial asthma for treatment, and investigated its clinical efficacy. The results showed that a significant difference existed between the treatment group and the control group, while the Pills can significantly reduce the serum IgE, improving one second forced expiratory gas volume and index of peak expiratory flow rate. Zhang et al. [57] using Gecko "Dingchuan Capsule" to treat 150 patients with bronchial asthma, and the clinical observation revealed that this Gecko formulation owns a good efficacy of relieving asthma. Zou et al. [58] again reported that Gecko "Dingchuan Capsules" exhibited excellent clinical effect of relieving asthma, expectorant, cease cough, anti-inflammation, immunity enhancement, antibacterial and anti-allergy, etc. while none of the toxic side effects have been found. Luan [59] described a large-scaled clinical investigation of Gecko "Dingchuan Capsules" upon chronic bronchitis and asthma patients for 206 cases, the treatment obtained satisfactory results with the total effective rate of $94.7 \%$.

\subsection{Cantharides}

Cantharides used in TCM indicates the dry body of Mylabris phalerata Pallas or Mylabris cichorii Linnaeus 
(Meloidae). TCM physicians often take the acupoint application of cantharidin powder sticking to the patient, make sticking parts congestion, blistering, which may play a similar role like moxibustion, that is the "Tian Zhi" (natural moxibustion), also called vesiculation therapy. This therapy is mainly used for psoriasis, neurodermatitis, joint pain etc.. Guo et al. [60] compared the clinical efficacies of Cantharides ointment (compound Mylabris made of cantharidin powder with cloves and cinnamon) and traditional medicine vesiculation treatment on bronchial asthma; 110 patients were randomly divided into Cantharides ointment group (55 cases) and traditional "Tian Zhi" (vesiculation therapy, made from white mustard seed, rhizoma corydalis, asarum, raw kansui) group (55 cases), sticking the corresponding drugs to the testee and to compare the clinical efficacy as well as the quality of life of the testee. The results showed that total effective rate of Cantharides ointment group was 94.55\%, higher than that of the traditional "Tian Zhi" (vesiculation therapy) group (82.27\%); their score improvements of activity limitation, asthma status, irritants original of reaction etc. are significantly better than those in the control group $(P<0.01$ or 0.05$)$, which displayed that the Cantharidin ointment treatment of bronchial asthma has a significant effect. Wu et al. [61] performed the clinical trial of applicator acupuncture of Cantharidin ointment treatment on 200 cases of asthma and chronic bronchitis patients. The results were satisfactory: in 200 cases were cured 65 cases (32.5\%); markedly effective in 63 cases, accounting for $31.5 \%$; improved in 62 cases, accounting for $31 \%$; ineffective in 10 cases, accounting for $5.0 \%$; the total effective rate was $95 \%$.

\subsection{Nidus Vespae}

In TCM Nidus vespae (Chinese name "Feng Chao") means the dry nests of wasp Polistes olivaceous (De Geer), Polistes japonicus Saussure or Parapolybia varia Fabricius [62]. Shen et al. [63] using the "Cough Rehabilitation Capsule" (made of ginseng, Placenta, Gecko, dogwood, Honeycomb, Oriental Arborvitae, orange peel, licorice, etc.) on the treatment of patients with chronic bronchitis for 209 cases, the total effective rate was $97.36 \%$. Fan et al. [64] using self-made "Hive Shegan Pulvis" (made from Nidus vespae and Belamcanda chinensis L. Redouté) in the treatment of bronchial asthma for 40 cases, of which 34 cases were markedly effective, 5 cases improved, and 1 case ineffective, the total effective rate was $97.5 \%$. Jia [65] reported that the application of the drying Nidus vespae powdered with a little Gecko powder on the treatment of senile asthma, each administration for $0.5 \mathrm{~g}, 3$ times per day, and received a satisfactory curative effect.

\subsection{Pearl}

Chinese medicine pearl mainly means the particulate matter formed by stimulation occurred on bivalves mantle of Pteria martensii Dunker (Pteriidae), Hyriopsis cumingii Lea (Unionidae), Cristaria plicata Leach (Anodontidae) etc.. According to Xu's clinical report [66], 275 cases of asthma patients were treated with "Zhubei Dingchuan Wan" (made from pearl and Fritillaria cirrhosa D. Don), the effective rate of the testees was $86.5 \%$. In the treatment of 84 cases of chronic bronchitis by "Zhubei Dingchuan Wan", the effective rate was $86.9 \%$, and exhibited a satisfactory long-term late result.

\section{DISCUSSION AND OUTLOOK}

The pathogenesis of asthma is full of complexity. Some scholars believe that asthma is induced by the balance disorder of body Th1/Th2 ( $T$ helper cells called Th cells which can be divided into Th1, Th2, etc.), since normal people take Th1 as the dominant cell, while patients with asthma tends to over-differentiate Th0 to Th2 in the body. This leads to a dominant Th2 cell status, thereof the Th1/Th2 imbalance is an important foundation of asthma [67]. Dong [68] pointed out that the key mechanisms of asthma might be attributed to the imbalance between Th1/Th2 cells, the reduction of the Th1-type cytokines IFN-C, and the increasing secrete of pro-allergic cytokines IL-4, IL-5, IL-13 of Th2 cells. Nevertheless, nowadays more and more scholars tend to present IL-13, IL-4 variation plays an important role in the occurrence of asthma [69-71]. Walsh's studies [72] suggested that among the various cells participated in airway inflammation such as eosinophils (EOS), macrophages, mast cells, T-lymphocytes cells played a key role, which is closely related to the severity of the diseases in both the degree of airway infiltration and the incidence of asthma. Qiu et al. [73] showed that the NF-KB system may induce the expression of GATA-3, thereby promoting dominant responses of Th2 cell, leading to airway inflammation.

This review summarizes animal drugs applied for the treatment of asthma or old chronic bronchitis. It could be found that Chinese pharmaceutical scientists has accumulated a wealth of experience in the clinical application, find fact that animal drugs were popularly used in the treatment of asthma or old chronic bronchitis. Not only the single dose of earthworm, 
scorpion, silkworm, ants, cantharidin, pillbugs, centipedes etc. can afford a satisfactory curative effect, but also the insect drug compatibility demonstrated significant efficacy [74]. Nevertheless, the experimental study of insect drugs on bronchial asthma intervention mechanism has just started and thereby is rarely reported. Therefore, further systematic pharmacological studies should be accomplished to determine the therapeutic effect of experienced animal drugs or insect drugs, which is urgently expected to provide a reliable basis for clinical trials of these new drug resources of asthma and old chronic bronchitis.

In addition, it is worth to mention that haze wether is a new phenomenon emerged in China in recent years. The possible negative influences arose by several large-scale outbreaks of haze on human health caused widespread concern in the community. The possible main cause of haze might be the high PM2.5 pollution, since PM2.5 pollution and the coerced chemical contaminants have a significant impact in varying degrees upon human and animal respiratory systems, cardiovascular systems, reproductive and nervous systems, while for the human suffering cancer is also associated responsibilities. Prevention of haze-caused respiratory diseases, especially in patients with asthma and chronic bronchitis caused by haze is a new but urgent theme [75].

Chinese National Development and Reform Commission has established National-local Joint Engineering Research Center of Entomoceutics, which recently performed systematic study of more than 1,300 species of extract and effective part of the medicinal insects. They found that a variety of insect extracts or effective parts can significantly inhibit the acute and chronic inflammation, and inhibit edema in rat smooth muscle relaxation in rodent animal experiments. In vitro experiments also revealed that more of the active candidates can enhance TNF- $\alpha$ and inhibit IL-2 etc. The research center is now concentrated on directional filtering of innovative drugs for anti-asthma from those which can effectively inhibit inflammation active parts from insects. Moreover, the chronic obstructive pulmonary disease (COPD) model using guinea pigs has been established for further animal experiments. The experimental results of effective parts of medicinal insects on the IL-4, IL-13, and interfere ability of Th1/Th2 ratio, as well as relevant clinical observation will be published soon. Although the international community has different views on alternative medicine and folk medicines, however, in line with internationally accepted standards and pharmacological study of the model, as well as the standard screening test program, the animal medicine in TCM, especially medicinal insects will remarkably promote the R\&D progress in the international fight against the chronic asthma and chronic bronchitis. Apparently, to produce such a landmark heavyweight drugs need collaborative innovation from multiple disciplines, multiple departments across multiple industries working together.

\section{ACKNOWLEDGEMENTS}

This work was supported by IRTSTYN (2010-ZY011), the P-MOST Programme for Yunnan Innovative Research Team, Key Projects of Science and Technology Development Plan of Yunnan Province 2012, 2011 Collaborative Innovation Center of Yunnan Province, and Special Funds for the Development of Strategic Emerging Industries Project in Yunnan Province 2013. We thank Prof. Dr. Joachim Stöckigt and Dr. Zhang Jingxin (Sinofi Co., USA) for the helpful discussions. We are in debt to Prof. Dr. Yang Zi-zhong (N-L JERCE) for the help on entomological taxonomy.

\section{REFERENCES}

[1] Li JS, Jia MR. Authentication of Chinese Materia Medica, Shanghai Scientific and Technical Publishers, Shanghai, China 1997; p. 575.

[2] Nanjing University of Chinese Medicine. The Dictionary of Chinese Materia Medica, Shanghai Scientific and Technical Publishers, Shanghai, China 1979; pp. 1-2754.

[3] Zeng MY, Zeng JF. Resource Records of Chinese Materia Medica, Science and Technology Publishing House of Beijing, Beijing, China 1994; p. 3.

[4] Masoli M, Fabian D, Holt S, Beasley R. The global burden of asthma: executive summary of the GINA dissemination committee report. Allergy 2004; 59: 469-78. http://dx.doi.org/10.1111/j.1398-9995.2004.00526.x

[5] Corrigan CJ, Kay AB. The roles of inflammatory cells in the pathogenesis of asthma and of chronic obstructive pulmonary disease. Am Rev Respir Dis 1991; 143: 1165-68. http://dx.doi.org/10.1164/ajrccm/143.5 Pt 1.1165

[6] Barnes PJ. Endogenous inhibitory mechanisms in asthma. Am J Respir Crit Care Med 2000; 161(2Suppl): S176-S181. http://dx.doi.org/10.1164/ajrccm.161.supplement 2.a1q4-6

[7] Ziment I, Tashkin DP. Alternative medicine for allergy and asthma. J Allergy Clin Immun 2000; 106: 603-14. http://dx.doi.org/10.1067/mai.2000.109432

[8] Wen DD, Wang M. Progress of Periostacum Cicadae and their combination treatment of asthma. Chin $\mathrm{J}$ Exper Trad Med Form 2012; 18: 242-45. http://dx.doi.org/10.1067/mai.2000.109432

[9] Chen RS. Chinese Pharmacopoeia Practical Manual, Jiangsu Science and Technology Press, Jiangsu, China 2004; pp. 40-42.

[10] Zhang MY. Study on effects of anti-bronchial asthma of Periostracum cicadae and pharmaco-mechanisms. Master Thesis, Hebei Medical University, Hebei, China 2007, pp. 164. 
[11] Xu SN, Zhang MY, Wang YM, Jin YR, Liu ZM. Antitussive, expectorant and antiasthmatic effects of periostracum cicadae. Chin Pharmacol Bull 2007; 23: 1678-89.

[12] Xu LR. Periostracum cicadae Zhike Granule clinical observation on the treatment of acute bronchitis. Trad Chin Med Res 1995; 8: 26-28.

[13] Hao LL, Liu SL, Zhang GJ. Research progress of traditional Chinese medicine on scorpion. Acta Chin Med Pharm 1994; 22: 49-52.

[14] Wang YP, Lü XR. Research progress of purification and pharmacological activities on scorpion venom of Buthus martensii Karsch. Chin Trad Herb Drugs 2000; 31: 59-61.

[15] Zhou Y, Yuan XF. Treatment of 62 cases children with cough variant asthma by Chinese traditional medicine. Liaoning $\mathrm{J}$ Trad Chin Med 1999; 26: 402.

[16] Li HY, Yang PL, Huang HY, Cui JG, Yang J, et al. Effects of scorpio-scolopendra on expressions of airway inflammation and airway remodeling in asthmatic rats. Chin J Exper Trad Med Formulae 2013; 19: 206-10. http://www.cnki.net/kcms/ detail/11.3495.R.20121032.1143.006.html

[17] Korideck H, Peterson JD. Noninvasive quantitative tomography of the therapeutic response to dexamethasone in ovalbumin-induced murine asthma. J Pharmacol Exper Ther 2009; 329: 882-89. http://dx.doi.org/10.1124/jpet.108.147579

[18] Li W, Xie H, Chen JH. Advances of pharmaceutical research on ant. Chin J Gerontol 1994; 14: 315-6.

[19] Zhang JH, Ma AH, Xie XJ, Li SL. The research status of dietotherapy on ant. Jiangsu J Trad Chin Med 1996; 17: 4345.

[20] Zhao Y, Wang Q, Li AY, Xie PS, Pan SJ. The experimental studies of effect of sedation, anti-inflammatory, liver protection, spasmolysis and toxicity by Polyrhachis vicina Roger. Guangxi J Trad Chin Med 1983; 6: 39.

[21] Chen GX, Yang JR, Ding BP, Xu Y, Chen B, et al. The research of pharmacodynamic and toxicity on Shedan Chenpi capsules. Chin Trad Patent Med 2000; 22: 810.

[22] Cai JY, Zhang YH, Ruan HL, P HF, Zhao W, et al. The comparative pharmacodynamic study between Shedan Chuanbei Pulvis and Shedan Hubei Pulvis. Chin Arch Trad Chin Med 2005; 23: 295-96.

[23] Zhang YM, Wu BD, Wang YZ, Zhang YJ. Clinical observation of admixture of snake bile and bamboo juice in treating 82 cases of infantile bronchitis. Acta Adad Med Suzhou 1998; 18: 306.

[24] Liu ZC, Guo XL, Zhang SQ. The curative effect observation of treatment of bronchitical asthma on ahylysantinfarctasely. Inner Mongolia Med J 1993; 13: 23.

[25] Lü XX, Fang JA. The application of human placenta with bronchial asthma in remission stage. Proc Clin Med 2001; 10: 622-23. http://d.wanfangdata.com.cn/Periodical_sxlcyy 200108049.aspx

[26] Jiang YA, Tao GS. The treatment of childhood asthma manipulation combined with human placenta. J Trad Chin Med 2002; 18: 21. http://d.wanfangdata.com.cn/ Periodical_amydy200204018.aspx

[27] Zhang J, Cui YM. Treatment of asthma by Bombyx batryticatus. J Trad Chin Med 2009; 50: 724.

[28] Yu J. Studies on the clinical application and modern pharmacological studies of Bombyx batryticatus. J China Trad Chin Med Inform 2010; 2: 185-86. http://d.wanfangdata. com.cn/Periodical_zgzyyzx201007174.aspx

[29] Li H. Treatment of 42 cases of cough variant asthma based on insect drugs. Jiangsu J Trad Chin Med 2005; 26: 19. http://d.wanfangdata.com.cn/Periodical_jszy200511010.aspx

[30] Liu H, Gao P, Wu X, Yang ZZ, Liu WH, et al. Utilization of Polislidae wasp venom as potential new insect drugs in the
R\&D of wellness industry. Intern J Wellness Indust 2012, 1: 241-49.

http://dx.doi.org/10.6000/1927-3037.2012.01.04.4

[31] Gao LJ, Wu J. Advance on the main compositions and the functions of honeybee venom. Genom Appl Biol 2013; 32 246-53.

http://dx.doi.org/10.3969/gab.032.000246

[32] Wang B. The separation and structural identification of major components in Yunnan Vespa venom. Master Thesis, Dali University, Yunnan, China 2014, pp. 1-81.

[33] Tang YJ, Qiu T, Xu ZB, Xu D, Xiao J, et al. The role of ERK $1 / 2$ in airway remodeling and smooth muscle proliferation in chronic obstructive pulmonary disease. Shaanxi Med J 2009; 38: 3-9.

[34] Xu AM, Liu QH, Zhou Y. The treatment of 50 cases of chronic bronchitis by traditional Chinese medicine. China Prac Med 2009; 4: 186. http://d.wanfangdata.com.cn/ Periodical_zgsyyy200922154.aspx

[35] Deng ML, Gao SX. Chinese Animal Drugs, The Peoples Press of Jilin, Jilin, China 1981, p. 54.

[36] Lin MX, Liu SM. The progress of clinical application of Armadillidiam vulgare. J Beijing Univ Trad Chin Med 2000; 12: $127-28$

[37] Wang QC, Liu GF. A new anti-thrombosis agent acutobin Chin J New Drugs 2000; 9: 270-72. http://d.wanfangdata. com.cn/Periodical_zgxyzz200004028.aspx

[38] Qin R. Treatment of 30 cases of chronic bronchitis by acutase. Chin Commun Doc 2000; 15: 24.

[39] Gao XM. Traditional Chinese Medicine, Chinese Press of Traditional Chinese Medicine, Bejing, China 2000, p. 480.

[40] Chen JB, Wang GZ, Chen GL, Wang CY. Study on the chemical ingredients in pheretima vulgaris. Chin Trad Patent Med 1997; 19: 35-36.

[41] Shan B, Wu JX, Zhang RY, Pan SJ. The research progress of pharmacological effects on earthworm. Med Res Educ 2009; 26: 77-80.

[42] $\mathrm{He} \mathrm{H}$, Li WP. The research of the main composition and role of the earthworm. J Ani Sci Veter Med 2008; 27: 28-30.

[43] Lü JS, Wu W, Meng DS, Chen L, Xiang MF. Study on antiinflammatory and analgesia effect of alcohol extract of Lumbricus. China Pharm 2003; 6: 16-18.

[44] Lin SQ, Zou $\mathrm{KH}$. Affecting tumorigenic mouse immune function and antioxidase of QY-I from earthworm. Strait Pharmaceut J 2002; 14: 10-12.

[45] Jin GR, Xu GZ. Effect of coagulation and fibrinolysis infarction role lumbrokinase. Chin J New Drugs Clin Remed 1999; 18: 48-50.

[46] Zeng XR, Zhang BY, Mai XX, Li XA, Zhang ZX, et al. The effects of extraction from earthworm on various tumour cells in vitro. J Shanxi Med Univ 1995; 26:[1],81-83.

[47] Zhou MM, Chu XP, Yang HZ, Kou F, Zhao AH, et al. Antiinflammatory and anti-allergic effects of acidic fraction of Pheratima extract in asthma mice induced by ovalbumin. China J Chin Mater Med 2008; 33: 2249-252.

[48] Wu SM, Zhang CP. The influence of lumbricus injection on pulmonary function in the rats with asthma. Shanxi Med $J$ 2009; 38: 511-13.

[49] Lin JH, Liu BY. The effect of traditional Chinese medicine on airway in asthmatic guinea pig. Shanghai Med J 1996; 19: 638-41.

[50] Yuan QS, Chen YG, Chen RB, Ming CR. Effects of Dilong Capsule on C-JUNmRNA expression in lung tissue of guinea pigs with asthma. Chin J Trad Med Sci Tech 2005; 12: 81-82.

[51] Qi HW, Li HZ, Liu YG, Lü KJ, Gao PS. The relationship between proto-oncogenes expression and airway inflammatory cell infiltration in asthma. Chin J Pathophysiol 2001; 17: I,293-96. 
[52] Ding N. Treatment of 40 cases with cough variant asthma by earthworm Erchen decoction. Shaanxi J Trad Chin Med 2010; 31: 943-44.

[53] Yang AM, Leng YX. Treatment of 71 cases of chronic bronchitis by Huoxue Tongluo decoction according to traditional Chinese medicine syndrome differentiation. Shaanxi J Trad Chin Med 2010; 31: 393-94.

[54] Zhu JY. Treatment of 100 patients with acute exacerbations of chronic bronchitis by Xiaoqinglong Decoction with earthworm. J New Chin Med 2003; 35: 56-57.

[55] Li H, Su Z. Study on pharmacodynamic and toxicology of Hajie Dingchuan dispersible tablets. J Med Forum 2008; 29: 11-13.

[56] Li P. The clinical research of Gejie Fangchuan Pills for bronchial asthma. J Emerg Trad Chin Med 2007; 16:10551056.

[57] Zhang LJ, Wu P, Du P. Clinical observation of Gejie Dingchuan Capsule on the treatment of 150 cases of bronchial asthma. Prac J Card Cereb Pneum Vasc Dis 2006; 14: 306.

[58] Zou JM, Zuo J, Li MZ, Li AH. Study on pharmacodynamic and toxicology of Gejie Dingchuan Capsule. Chin Trad Herb Drugs 2003; 34: 343-46.

[59] Luan Y. Clinical observation of Gejie Dingchuan capsule on the treatment of 206 cases of chronic bronchitis and bronchial asthma. China Pract Med 2009; 4: 170.

[60] Guo XQ, Fu RY, Zhu Y, Li Y, Wang YL, et al. The clinical observation of compound mylabris extract with acu-point application for bronchial asthma. J Emerg Trad Chin Med 2013; 22: 1954-55. http://d.wanfangdata.com.cn/Periodical_ zgzyjz201311068.aspx

[61] $\mathrm{Wu} \mathrm{AL}, \mathrm{Wu} X \mathrm{X}, \mathrm{Xu} \mathrm{HF}$. The treatment 200 cases of compound mylabris extract with acu-point application control bronchial asthma and chronic bronchitis. Chin Acupunct Moxibus 1999; 19: 140-42.

[62] Wang B, Zhang CG, Gao PF, Wu XM, Zhao Y. Research progress in nidus vespae, a traditional Chinese medicine derived from in insects. J Pharm Sci Innov 2013; 2: 1-9. http://dx.doi.org/10.7897/2277-4572.02680

[63] Shen QL, Jing HG, Li KD, Gu Y, Li ZR. The treatment 152 cases of chronic bronchitis by Cough Rehabilitation Capsule (Kechuan Kangfu Capsule). J Chengdu Univ TCM 1998; 21: 22-25.
[64] Fan J, Fu P, Fu XM. The clinical observation of Fengfang Shegan powder for asthma. Hubei J Trad Chin Med 2004; 26: 32 . http://d.wanfangdata.com.cn/Periodical_hbzyzz 200401019.aspx

[65] Jia $X Y$. The experience of clinical application of nidus vespae. Res Trad Chin Med 2000; 16: 31.

[66] Xu ZR. The curative effect analysis in 58 cases of bronchial asthma and chronic bronchitis treated by Zhubei Dingchuan Wan. Guangdong Med J 1987; 8: 54.

[67] Cooper MA, Fehniger TA, Caligiuri MA. The biology of human natural killer-cell subsets. Trends Immun 2001, 22 : 633-40. http://dx.doi.org/10.1016/S1471-4906(01)02060-9

[68] Dong ZQ. The mechanism and treatment of allergic asthma in children. Pediat Emerg Med 2000; 15: 174-76.

[69] Prieto J, Lensmar C, Roquet A, van der Ploeg I, Gigliotti D, et al. Increased interleukin-13 mRNA expression in bronchoalveolar lavage cells of atopic patients with mild asthma after repeated low-dose allergen provocations. Respir Med 2000; 94: 806-14.

\section{http://dx.doi.org/10.1053/rmed.2000.0826}

[70] Walter DM, McIntire JJ, Berry G, McKenzie ANJ, Donaldson, $\mathrm{DD}$, et al. Critical role for $\mathrm{IL}-13$ in the development of allergen-induced airway hyperreactivity. J Immun 2001; 167 4668-675

http://dx.doi.org/10.4049/jimmunol.167.8.4668

[71] Kumar RK, Herbert C, Yang M, et al. Role of interleukin-13 in eosinophil accumulation and airway remodelling in a mouse model of chronic asthma. Clin Exp Allergy 2002; 32: 1104111. http://dx.doi.org/10.1046/j.1365-2222.2002.01420.x

[72] Walsh GM. Eosinophil apoptosis: mechanisms and clinical relevance in asthmatic and allergic inflammation. Brit $J$ Haemat 2000; 111: 61-67.

http://dx.doi.org/10.1111/j.1365-2141.2000.02103.x

[73] Qiu LQ, Cresswell P, Chin KC. Viperin is required for optimal Th2 responses and T-cell receptor-mediated activation of NF-kB and AP-1. Blood 2009; 113: 3520-529. http://dx.doi.org/10.1182/blood-2008-07-171942

[74] Liu L. The treatment of 98 cases of persistent cough mainly by worm medicine. J New Chin Med 2007; 39: 52-53.

[75] Chen RJ, Kan HD. Haze/fog and human health: a literature review. Chin J Nat 2013; 35: 342-44. http://dx.doi.org/10.3969/issn.0253-9608.2013.05.006

\section{DOI: http://dx.doi.org/10.6000/1927-3037.2014.03.02.5}

(C) 2014 Wu et al.; Licensee Lifescience Global.

This is an open access article licensed under the terms of the Creative Commons Attribution Non-Commercial License (http://creativecommons.org/licenses/by-nc/3.0/) which permits unrestricted, non-commercial use, distribution and reproduction in any medium, provided the work is properly cited. 